\title{
Drift-flux model for gas-liquid flow subjected to centrifugal fields
}

\author{
Marcos Penteado ${ }^{1}$, Saon Vieira ${ }^{2}$, Marcelo Castro $^{3}$, and Antonio Bannwart ${ }^{4}$ \\ ${ }^{1}$ Universidade Estadual de Campinas Centro de Estudos de Petróleo \\ ${ }^{2}$ Petrobras \\ ${ }^{3}$ Universidade Estadual de Campinas Centro de Estudos de Petroleo \\ ${ }^{4}$ Universidade Estadual de Campinas
}

March 11, 2021

\begin{abstract}
Centrifugal pumps are used in several industrial processes. It is common the operation of this equipment with gas-liquid mixtures, which is the case of the electrical submersible pumping artificial lift method used in the oil industry. The increase of free gas fraction inside the pump may lead to unstable operation and problems such as surging and gas locking phenomena to occur. In this study a drift-flux model is proposed for the gas-liquid flow subjected to centrifugal fields using the impeller as an example. The model is closed with experimental data of bubble diameter, displacements and velocities acquired via high-speed camera at several different rotational speeds and gas mass flow rates using water as the continuous medium. From the modeling and the forces balance in the bubbles, a quantitative criterion for the start point of surging and gas locking conditions was proposed.
\end{abstract}

\section{Hosted file}

Main.pdf available at https://authorea.com/users/400808/articles/513083-drift-flux-modelfor-gas-liquid-flow-subjected-to-centrifugal-fields 